centralisation of cancer services to ensure delivery of an effective management plan to all patients diagnosed with gastrooesophageal cancer. With the high mortality, monitoring and auditing treatment outcomes is vital in ensuring that these patients receive the best available treatment. The recent publication of the National Oesophago-Gastric Cancer Audit data further re-iterates this and forms the crux of evidence-based management of patients diagnosed with gastro-oesophageal cancer.

Methods The study aims to measure the survival outcome of patients diagnosed with both gastric and oesophageal cancer in our NHS trust who has a catchment population of 500000 across the 2 district general hospitals. We retrospectively reviewed the medical notes, computerised notes of the multidisciplinary team (MDT) meetings as well as endoscopy reports of all patients diagnosed with gastro-oesophageal cancer in the 5-year period from 1 January 2004 to 31 December 2008. The date of death was noted and the number of survivors as of 15 July 2010 were taken into account.

Results 234 and 139 patients were diagnosed with oesophageal and gastric cancer respectively, of which data from 6 patients were unavailable. As of 15 July 2010, 46 (12\%) of all patients traced are still alive, 31 (13\%) with oesophageal cancer and 15 (11\%) with gastric cancer. For those that have died, the median survival was 156 days (2-1594) and 251 days (4-1413) for patients with gastric and oesophageal cancer respectively. For patients with oesophageal cancer, the median survival in those managed palliatively was 247 days (4-1022) while the median survival was 383 days (71-1413) in those that had neoadjuvant chemotherapy and surgery. Survival rates are shown below.

Conclusion The improved survival after treatment with curative intent (surgery \pm neoadjuvant chemotherapy or primary chemoradiotherapy) is encouraging. For oesophageal cancer, our 1-year survival rate $(75 \%)$ in this group mirrors the results from the recently published National Oesophago-Gastric Cancer Audit 2010 (76.1\%). There are more patients with gastric cancer being managed palliatively and this is reflected by the lower survival rates in this group. Centralisation of services in this trust occurred in 2009 and comparison with these data in future are vital in monitoring its success.

Table 1 PWE-114 Survival in gastro-oesophageal cancer

PWE-114 LONG TERM SURVIVAL IN GASTRO-0ESOPHAGEAL CANCER

doi:10.1136/gut.2011.239301.377

SF Neong, 1,* J Deacon, ${ }^{1}$ I R Sargeant, 1 D L Morris² 'Gastroenterology, Lister Hospital, Stevenage, UK; ${ }^{2}$ Gastroenterology, Queen Elizabeth II Hospital, Welwyn Garden City, UK

Introduction One of the changes implemented following the publication of the Improving Outcome Guidances (IOG) is the

\begin{tabular}{llll}
\hline Gastric cancer & $\begin{array}{l}\text { \% Overall } \\
\text { survival }\end{array}$ & $\begin{array}{l}\text { \% Survival with } \\
\text { curative intent }\end{array}$ & $\begin{array}{l}\text { \% Survival with } \\
\text { palliative intent }\end{array}$ \\
\cline { 2 - 4 }$(\mathbf{n = 1 2 3})$ & $(\mathbf{n = 3 3 )}$ & $(\mathbf{n = 9 0 )}$ \\
\hline 6 months & 50 & 64 & 43 \\
12 months & 30 & 53 & 18 \\
42 months & 12 & 29 & 0 \\
Oesophageal cancer & $(\mathbf{n = 1 9 7 )}$ & $(\mathbf{n}=\mathbf{4 4 )}$ & $(\mathbf{n}=\mathbf{1 5 3 )}$ \\
6 months & 65 & 87 & 55 \\
12 months & 42 & 75 & 27 \\
42 months & 15 & 41 & 0 \\
\hline
\end{tabular}




\section{BSG abstracts}

Competing interests None.

Keywords 1-year survival, endoscopic therapy, gastrooesophageal cancer survival, treatment intent.

\section{REFERENCES}

1. National Oesophago-Gastric Cancer Audit 2010. Third Annual Report. 